\title{
Charakterisierung von CMOS RF Blöcken mittels Volterra-Reihen zur Optimierung des Designprozesses
}

\author{
B. Fei, A. H. Darrat, and W. Mathis \\ Leibniz Universität Hannover, Institut für Theoretische Elektrotechnik, Appelstr. 9A, 30167 Hannover, Germany
}

\begin{abstract}
Zusammenfassung. Im Rahmen dieser Arbeit werden die Volterra-Reihen zur Analyse der Nichtlinearität in RF Schaltungen verwendet, um den Designprozess für RF Systeme zu optimieren. Die auf Volterra-Reihen basierende Nichtlinearitätsanalyse wurde in eine Matlab-Toolbox implementiert. Diese Toolbox kann mittels Volterra-Reihen die symbolische Berechnung der Nichtlinearitätsparameter (harmonische Verzerrungen und Intermodulationsverzerrungen) eines RF Blocks für eine gegebene Architektur und Technologie durchführen. Danach können die symbolische Ausdrücke der Nichtlinearitätsparameter in Abhängigkeit von den Architekturparametern und Technologieparametern erhalten werden. Dies ermöglicht die Beschränkung der Wertebereiche der Architekturparameter und die Überprüfung auf die Erfüllung der Nichtlinearitätsspezifikationen für unterschiedliche Kombinationen von Architekturen und Technologien. Somit ist eine Beschränkung der Klassen der Architekturen und Technologien möglich. Die Toolbox wurde zur Verdeutlichung auf einen Low Noise Amplifier (LNA) der Inductive Source Degeneration (ISD) Architektur angewandt. Zur Verifikation wurde diese LNA-Schaltung auch in Cadence SpectreRF Design Tool simuliert.
\end{abstract}

\section{Einleitung}

Bei einem Design Flow eines RF Systems (siehe Abb. 1) erzeugt einerseits der Systemdesigner aus den Systemspezifikationen die Spezifikationen für die einzelnen Systemblöcke. Anderseits versucht der Schaltungsdesigner Schaltungen zu entwerfen, die die Blockspezifikationen erfüllen. Die meisten Schaltungsdesigner nutzen die Taylorreihen um die Nichtlinearität zu analysieren. Diese Methode ist allerdings nur für statische Schaltungen geeignet. In der Regel

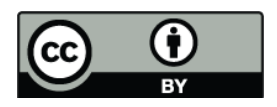

Correspondence to: A. H. Darrat (darrat@tet.uni-hannover.de) kann der Schaltungsdesigner zur Analyse der Nichtlinearität in Analogschaltungen numerische Methoden nutzen, z.B. numerische Integration und Harmonic Balance. Der Nachteil von numerischen Methoden ist, dass die Ergebnisse keine qualitativen Aussagen über die Abhängigkeit der Nichtlinearität mit den Schaltungsparametern ermöglichen. Das bedeutet, dass der Schaltungsdesigner mit solchen numerischen Methoden nicht erkennen kann, von welchen Schaltungsparametern die Nichtlinearität abhängt und wie diese Parameter sich auf die Nichtlinearität auswirken. Solche wertvolle Informationen können durch die symbolische Berechnung mit der Anwendung der Volterrareihen erhalten werden. Die Volterrareihen eignen sich für die Beschreibung von dynamischen schwach nichtlinearen Systemen und sind somit für die Beschreibung der RF Blöcke auch gut geeignet (Schetzen, 1980; Zadeh, 1953). Die symbolische Berechnung von den Nichtlinearitätsparametern ermöglicht die Charakterisierung der Blockspezifikationen für die Nichtlinearität in Abhängigkeit von den Schaltungsparametern.

Abbildung 2 beschreibt den vorgeschlagenen Ansatz. Die Nichtlinearitätsparameter eines RF Blocks, z.B. LNA, können mittels Volterra-Reihen in Abhängigkeit von den Architekturparametern und Technologieparametern beschrieben werden. Auf diese Weise können die Nichtlinearitätsparameter bei Festlegung der Architektur für verschiedene Technologien verglichen werden. Bei Festlegung sowohl der Architektur als auch der Technologie können die Nichtlinearitätsparameter gegen mehrere Architekturparameter graphisch dargestellt werden. Weiterhin können die Wertebereiche der Architekturparameter, die die Blockspezifikationen erfüllen, für die bestimmte Architektur und Technologie beschränkt werden. Somit kann der Schaltungsdesigner bestimmen, welche Kombination von der Architektur und Technologie mit welchen Wertebereichen der Architekturparameter die Blockspezifikationen am besten erfüllen. 


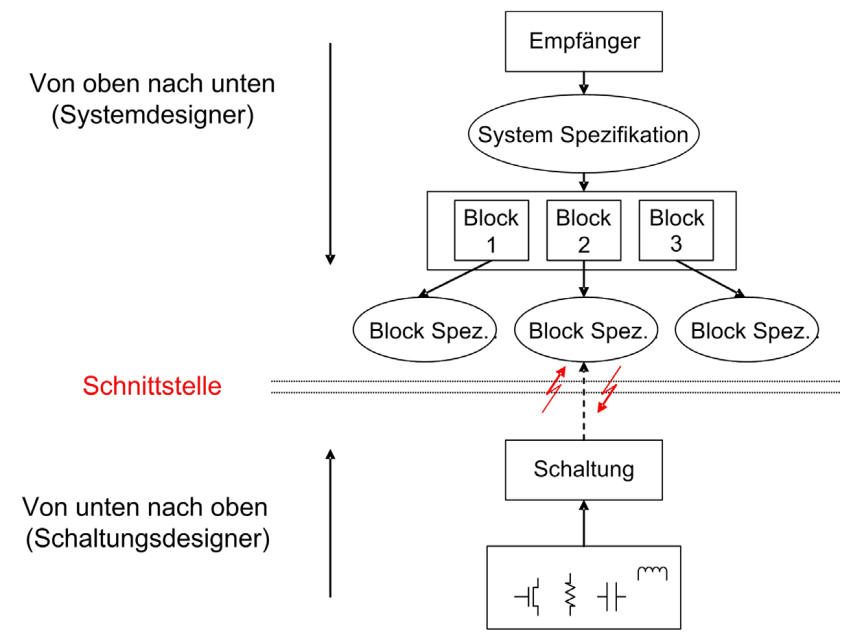

Abb. 1. Design Flow von RF Systemen.

Dieser Ansatz wird in einer symbolischen Design-Toolbox implementiert. In der Toolbox werden die Taylorreihen zur Ermittlung der Nichtlinearitätskoeffizienten verwendet. Compacted Modified Nodal Analysis (CMNA) (Gielen et al., 1989; Gielen und Sansen, 1991) wurde zur Bestimmung des Gleichungssystems verwendet. Diese Toolbox führt zu einem graphischen Optimierungsprozess, der eine parametrische Analyse ermöglicht. Davon werden die graphischen Darstellungen der Nichtlinearitätsparameter gegen mehrere Architekturparameter erzeugt.

\section{Volterra-Reihen}

Die Volterrareihen beschreiben die Beziehung zwischen dem Eingang und dem Ausgang eines dynamischen nichtlinearen zeitinvarianten Systems in die folgende Form (Schetzen, 1980):

$$
y(t)=\mathcal{H}_{1}[x(t)]+\mathcal{H}_{2}[x(t)]+\ldots+\mathcal{H}_{n}[x(t)]+\ldots
$$

mit

$$
\begin{aligned}
\mathcal{H}_{n}[x(t)]= & \int_{-\infty}^{+\infty} \ldots \int_{-\infty}^{+\infty} h_{n}\left(\tau_{1}, \ldots, \tau_{n}\right) x\left(t-\tau_{1}\right) \\
& \ldots x\left(t-\tau_{n}\right) d \tau_{1} \ldots d \tau_{n}
\end{aligned}
$$

wobei $\mathcal{H}_{n}[x(t)]$ ein mehrdimensionales Faltungsintegral repräsentiert und Volterraoperator n-ter Ordnung genannt wird. $h_{n}\left(\tau_{1}, \ldots, \tau_{n}\right)$ in Gleichung (2) ist als mehrdimensionale Impulsantwort definiert und wird Volterrakern n-ter Ordnung genannt.

Für ein schwach nichtlineares System sind nur die ersten drei Ordnungen der Volterra-Reihen zu berücksichtigen. Die harmonischen Verzerrungen (HD) und die Intermodulationsverzerrungen (IM) lassen sich in Abhängigkeit von den

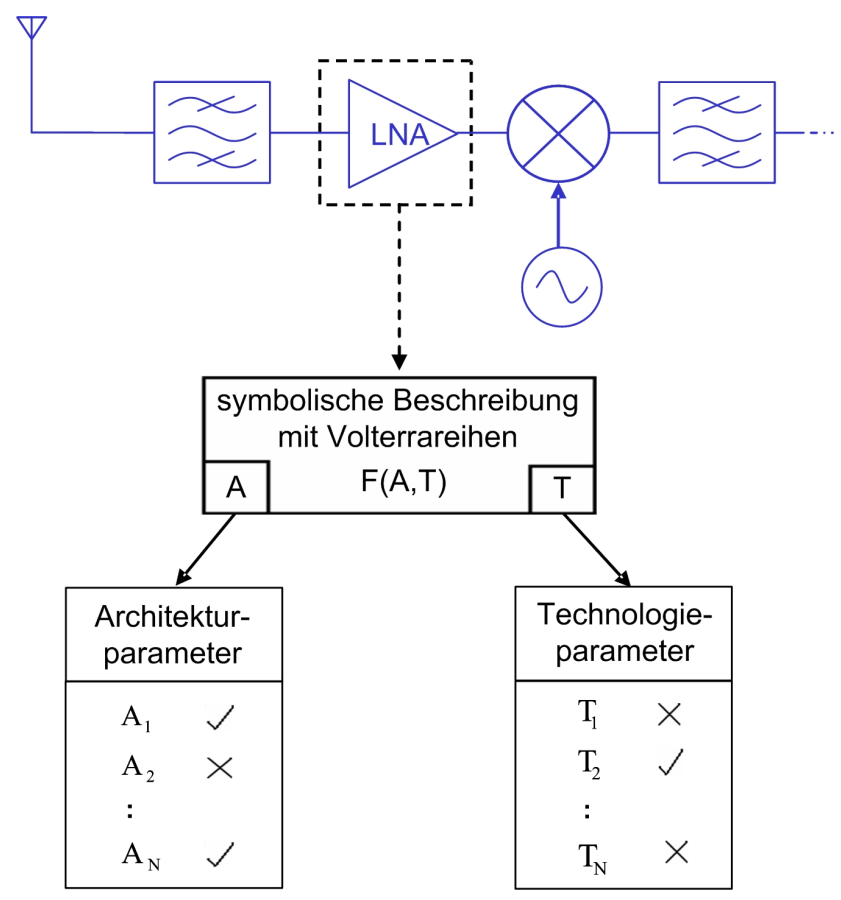

Abb. 2. Charakterisierung eines RF Blocks in Abhängigkeit von Architektur- und Technologieparametern.

Fouriertransformation der Volterrakerne darstellen. Die Ausdrücke von $\mathrm{HD}_{2}, \mathrm{HD}_{3}, \mathrm{IM}_{2}$ und $\mathrm{IM}_{3}$ sind (Wambcaq und Sansen, 1998):

$$
\begin{aligned}
& H D_{2}=\frac{A_{1}}{2}\left|\frac{H_{2}\left(j \omega_{1}, j \omega_{1}\right)}{H_{1}\left(j \omega_{1}\right)}\right| \\
& H D_{3}=\frac{A_{1}^{2}}{4}\left|\frac{H_{3}\left(j \omega_{1}, j \omega_{1}, j \omega_{1}\right)}{H_{1}\left(j \omega_{1}\right)}\right| \\
& I M_{2}=A_{1}\left|\frac{H_{2}\left(j \omega_{1}, \pm j \omega_{2}\right)}{H_{1}\left(j \omega_{1}\right)}\right| \\
& I M_{3}=\frac{3}{4} A_{1}^{2}\left|\frac{H_{3}\left(j \omega_{1}, \pm j \omega_{2}, \pm j \omega_{2}\right)}{H_{1}\left(j \omega_{1}\right)}\right|
\end{aligned}
$$

\section{Bestimmung der Volterrakerne}

Um die Volterrakerne zu bestimmen müssen zunächst die Nichtlinearitätskoeffizienten ermittelt werden. Die Nichtlinearitätskoeffizienten einer Grundnichtlinearität können durch Taylorreihenentwicklung der Grundnichtlinearität ermittelt werden. Die Nichtlinearitätskoeffizienten entsprechen den Taylorkoeffizienten. Die Nichtlinearitätskoeffizienten jeder Grundnichtlinearität in der Schaltung werden zur Ermittlung der nichtlinearen Stromquellen benötigt.

Die Volterrakerne können ermittelt werden, indem das lineare Gleichungssystem der linearisierten äquivalenten 
Schaltung in jeder Ordnung gelöst wird (Wambcaq und Sansen, 1998). Diese Methode wird bei dieser Arbeit modifiziert. Das lineare Gleichungssystem wird nur einmalig mittels Compacted Modified Nodal Analysis (CMNA) erzeugt. Die CMNA ergibt das Gleichungssystem der linearisierten äquivalenten Schaltung in die folgende Form:

$\mathbf{Y}(s) \cdot \mathbf{H}(s)=\mathbf{I N}$,

wobei $\mathbf{Y}(s)$ die Admittanzmatrix ist. $\mathbf{H}(s)$ ist der Vektor der Volterrakerne, die den Knotenspannungen entsprechen. IN ist der Vektor der Anregungen i-ter Ordnung, der aus einer externen Anregung und den nichtlinearen Stromqullen besteht. Die modifizierte Methode zur Bestimmung der Volterrakerne erster, zweiter und dritter Ordnung kann wie folgt zusammengefasst werden:

1. Das Gleichungssystem wird symbolisch gelöst. Danach wird ein Vektor von den Spannungen an jedem Knoten erhalten. Dieser Spannungsvektor ist eine Funktion von Schaltungsparametern (inklusive der externen Anregung und der nichtlinearen Stromquellen).

2. Die Variablen für die nichtlinearen Stromquellen im Spannungsvektor werden durch null ersetzt und die Frequenzvariable $s$ wird durch $s_{1}$ ersetzt. Die Volterrakerne erster Ordnung entsprechen dem Quotient aus dem neuen Spannungsvektor durch die Anregung.

3. Zur Bestimmung der Volterrakerne zweiter Ordnung wird die Anregung im Spannungsvektor durch null und die Frequenzvariable $s$ durch $\left(s_{1}+s_{2}\right)$ ersetzt. Die Variablen für die nichtlinearen Stromquellen bleiben. Danach werden die nichtlinearen Stromquellen zweiter Ordnung mit den Nichtlinearitätskoeffizienten zweiter Ordnung und den Volterrakernen erster Ordnung ermittelt. Nach dem Ersetzen der Variablen für die nichtlinearen Stromquellen durch die ermittelten nichtlinearen Stromquellen zweiter Ordnung können die Volterrakerne zweiter Ordnung erhalten werden.

4. Die gleiche Vorgehensweise wie im Schritt 3 zur Bestimmung der Volterrakerne zweiter Ordnung wird zur Bestimmung der Volterrakerne dritter Ordnung verwendet. Man beachtet hierbei, dass die Frequenzvariable $s$ durch $\left(s_{2}+s_{2}+s_{3}\right)$ ersetzt wird. Die nichtlinearen Stromquellen dritter Ordnung werden mit den Nichtlinearitätskoeffizienten zweiter und dritter Ordnung und den Volterrakernen erster und zweiter Ordnung ermittelt.

\section{Symbolische Toolbox}

Die Struktur der in Matlab entwickelten Toolbox wird in Abb. 3 gezeigt. Der Kern der Toolbox ist der Block "Volterraanalyse", in dem die oben genannte Methode zur Bestimmung der Volterrakerne implementiert wird. Die Eingangsdaten der "Volterraanalyse" sind das Gleichungssystem und

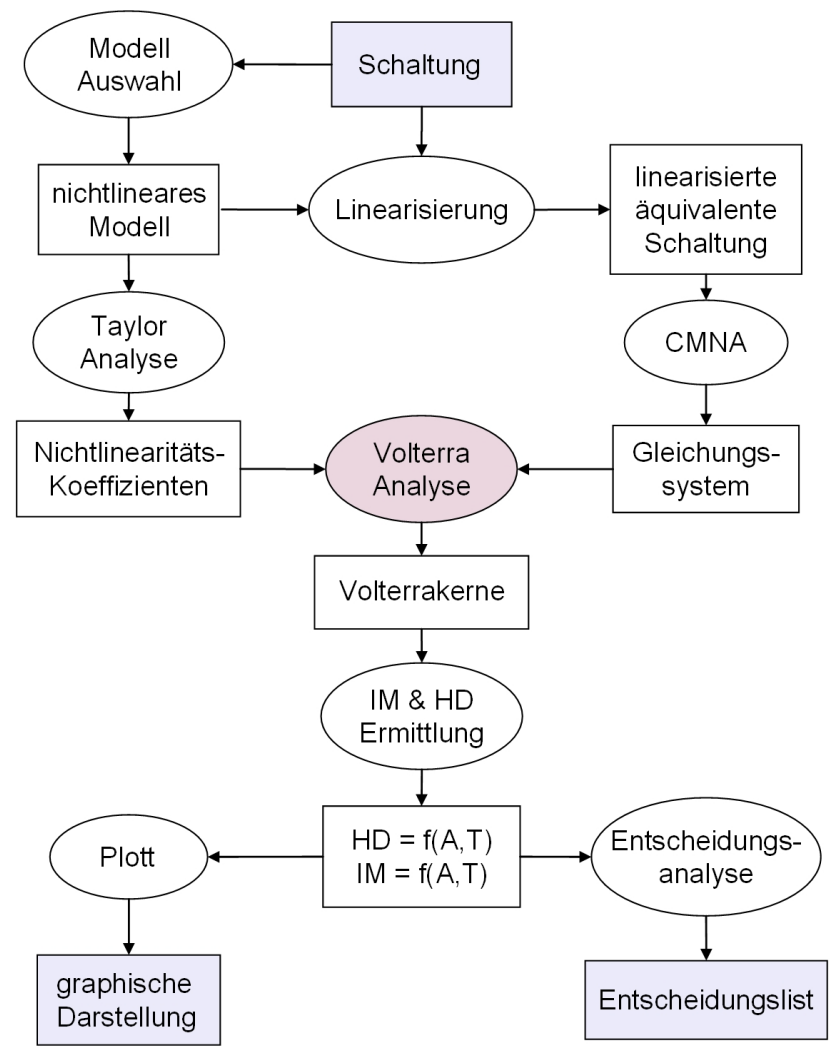

Abb. 3. Struktur der Toolbox.

die Nichtlinearitätskoeffizienten. Nach der "Volterraanalyse" werden die Volterrakerne erster, zweiter und dritter Ordnung bestimmt. Anschließend werden damit die Nichtlinearitätsparameter in Abhängigkeit von den Architekturparametern und Technologieparametern ermittelt. Danach einerseits können die Nichtlinearitätsparameter durch Plotten auf verschiedene Weisen dargestellt werden, andererseits kann durch eine Entscheidungsanalyse eine Entscheidungsliste automatisch erzeugt werden. Diese Entscheidungsliste zeigt für die ausgewählte Architektur und Technologie die Wertebereiche der Architekturparameter, die die Spezifikationen erfüllen.

Da die graphische Darstellung eines Parameters gegen mehr als drei Variablen nicht möglich ist, müssen die Werte von einigen der Architekturparameter festgelegt werden, um die graphische Darstellung zu ermöglichen. Die Anzahl der Architekturparameter kann durch die Betrachtung anderer Spezifikationsparameter außer den der Nichtlinearität reduziert werden. Hierbei muss allerdings die Priorität der anderer Spezifikationsparameter gegenüber den Nichtlinearitätsparametern beachtet werden. Wenn die Anzahl der Architekturparameter nach der Erfüllung der anderen Spezifikationen zu drei oder weniger reduziert wird, können die Nichtlinearitätsparameter gegen die gebliebenen Architekturparameter graphisch dargestellt werden. 


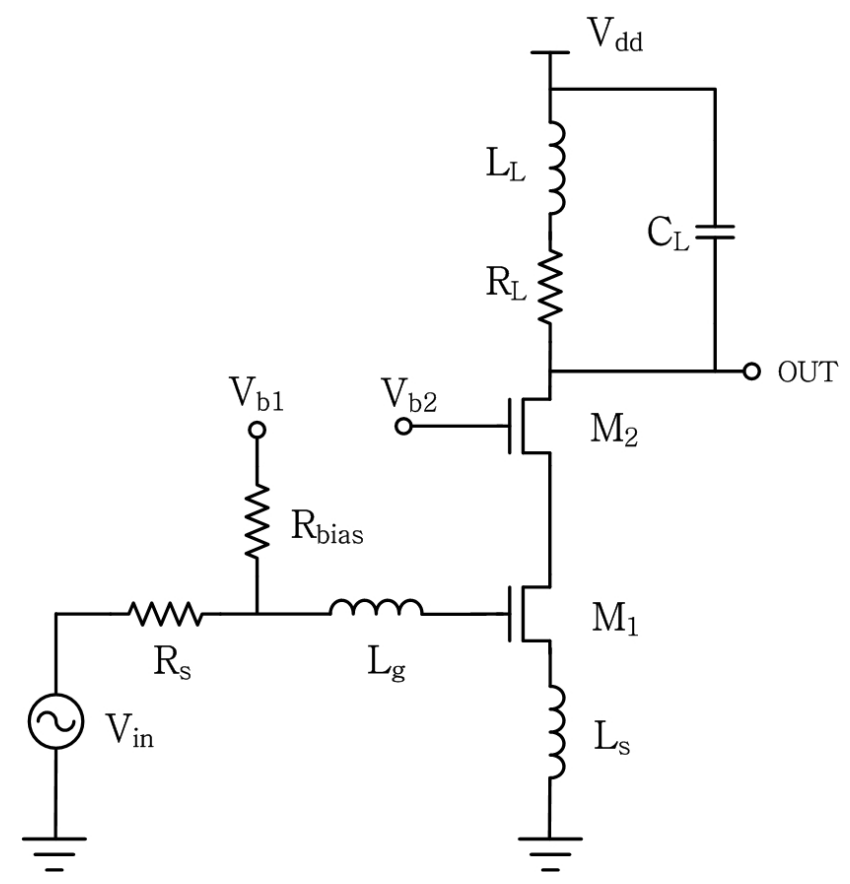

Abb. 4. CMOS LNA der Inductive Source Degeneration Architektur.

\section{Beispielschaltung}

Um die vorgeschlagene Prozedur zu verdeutlichen, wird diese Toolbox für die Analyse der Nichtlinearität eines CMOS LNAs der ISD Architektur (siehe Abb. 4) verwendet.

\subsection{MOSFET Modell}

In diesem LNA sind die nichtlinearen Bauelemente nur zwei MOSFET's. Für beide Transistoren wird das folgende Modell verwendet (Razavi, 2001):

$I_{D}=k_{n} \frac{V_{O D}^{2}}{1+\beta\left(V_{O D}\right)}$

mit

$k_{n}=\frac{1}{2} \mu_{0} C_{o x} \frac{W}{L}, \quad \beta=\frac{\mu_{0}}{2 v_{s a t} L}+\theta$,

wobei $W$ und $L$ jeweils die Kanalbreite und die Kanallänge sind. $C_{o x}$ ist die Silizium Oxid Kapazität pro Flächeneinheit und $\mu_{0}$ ist die Oberflächenbeweglichkeit. $v_{\text {sat }}$ ist die Sättigungsgeschwindigkeit und $\theta$ bezeichnet die Degradierung der Trägerbeweglichkeit. $V_{O D}$ ist die Overdrive-Spannung $\left(V_{G S}-V_{T H}\right)$.

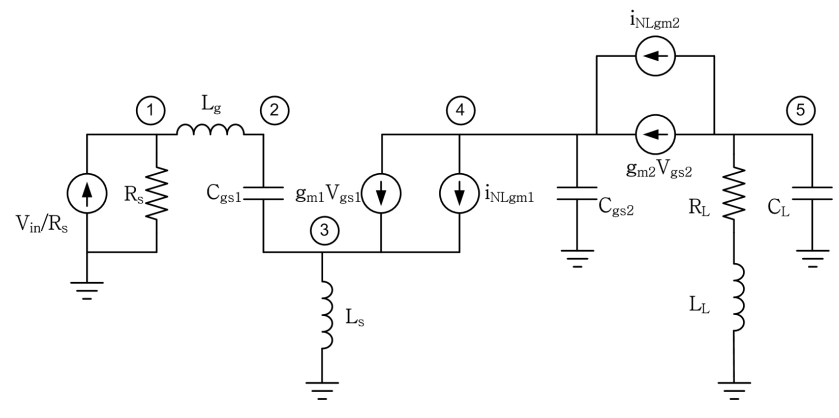

Abb. 5. Linearisierte äquivalente Schaltung vom ISD CMOS LNA.

Die Nichtlinearitätskoeffizienten erster, zweiter und dritter Ordnung können durch eine Taylorreihenentwicklung rund um den Arbeitspunkt $\left(V_{o d}, I_{d}\right)$ erhalten werden. Es folgt:

$g_{m}=\left.\frac{\partial I_{D}}{\partial V_{O D}}\right|_{V_{O D}=V_{o d}}=k_{n} V_{o d} \frac{2+\beta V_{o d}}{\left(1+\beta V_{o d}\right)^{2}}$

$K_{2 g_{m}}=\left.\frac{1}{2} \frac{\partial^{2} I_{D}}{\partial V_{O D}^{2}}\right|_{V_{O D}=V_{o d}}=k_{n} \frac{1}{\left(1+\beta V_{o d}\right)^{3}}$

und

$K_{3 g_{m}}=\left.\frac{1}{6} \frac{\partial^{3} I_{D}}{\partial V_{O D}^{3}}\right|_{V_{O D}=V_{o d}}=-k_{n} \frac{\beta}{\left(1+\beta V_{o d}\right)^{4}}$.

Für die Gate-Source Kapazität $C_{g s}$ gilt im Sättigungsbereich (Wambcaq und Sansen, 1998):

$C_{g s}=\frac{2}{3} C_{o x} W L$.

\subsection{Gleichungssystem}

Abbildung 5 zeigt die linearisierte äquivalente Schaltung vom ISD CMOS LNA. Dabei stellen $i_{\mathrm{NLgm} 1}$ und $i_{\mathrm{NLgm} 2}$ jeweils die nichtlinearen Stromquellen für die MOSFET's $M_{1}$ und $M_{2}$ dar. Die Spannungsquelle $V_{i n}$ als Anregung wird in eine Stromquelle umgewandelt, um die Größe der CMNAMatrix zu verkleinern. Nach der Verwendung der CMNA auf diese linearisierte Schaltung kann das Gleichungssystem be- 
stimmt werden. Es gilt:

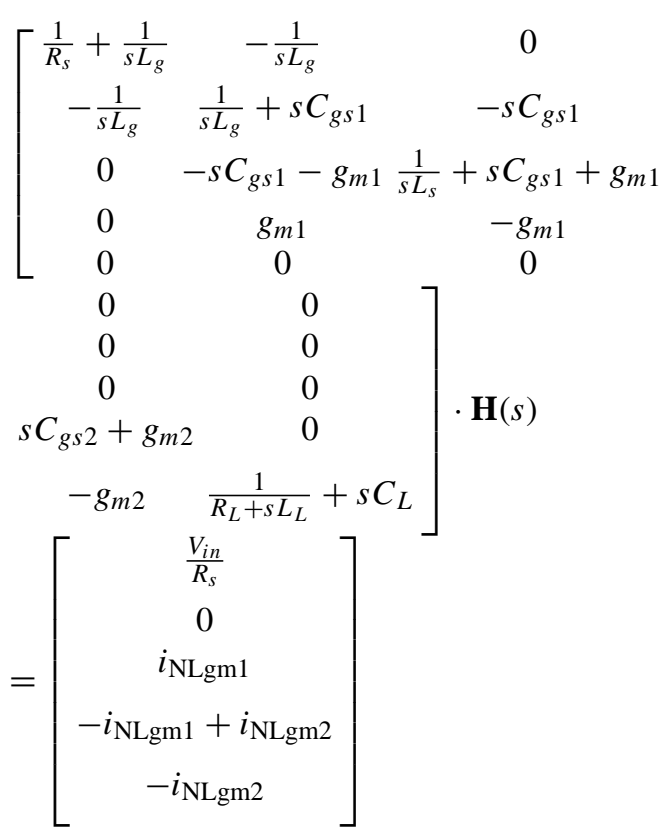

\subsection{Ermittlung der Volterrakerne und Nichtlinearitätsparameter}

Nach Ermittlung der Nichtlinearitätskoeffizienten und des Gleichungssystems kann die Volterraanalyse automatisch durchgeführt werden. Danach können die Volterrakerne erster, zweiter und dritter Ordnung in der folgenden Form erhalten werden:

$H_{\text {out } 1}\left(s_{1}\right)=f_{1}\left(P_{A}, P_{T}, s_{1}\right)$,

$H_{\text {out } 2}\left(s_{1}, s_{2}\right)=f_{2}\left(P_{A}, P_{T}, s_{1}, s_{2}\right)$

und

$H_{\text {out } 3}\left(s_{1}, s_{2}, s_{3}\right)=f_{3}\left(P_{A}, P_{T}, s_{1}, s_{2}, s_{3}\right)$,

wobei $P_{A}$ die Architekturparameter: $C_{L}, L_{g}, L_{L}, L_{s}, R_{L}$, $R_{S}, V_{o d 1}, V_{o d 2}, W_{1}$ und $W_{2}$ darstellt. $P_{T}$ stellt die Technologieparameter: $L, C_{o x}, \theta, \mu_{0}$ und $v_{\text {sat }}$ dar. Da die Längen der symbolischen Ausdrücke der Volterrakerne sehr groß sind, werden sie hier nicht gezeigt. Nach Bestimmung der Volterrakerne können die symbolischen Ausdrücke von $\mathrm{HD}_{2}, \mathrm{HD}_{3}$, $\mathrm{IM}_{2}$ und $\mathrm{IM}_{3}$ in Abhängigkeit von $A$ (Amplitude des Eingangssignals; bei Doppelfrequenz-Anregung wird angenommen, dass beide Amplituden gleich sind), $P_{A}, P_{T}$ und der Frequenz ermittelt werden. Die Interceptpunkte zweiter und dritter Ordung $\mathrm{IIP}_{2}$ und $\mathrm{IIP}_{3}$ sind Funktionen von $P_{A}, P_{T}$ und der Frequenz.

Nach Festlegung der Klasse der Technologie sind die Technologieparameter $P_{T}$ bekannt. Einige der Architekturparameter $C_{L}, L_{g}, L_{L}, L_{s}, R_{L}, R_{s}$ und $W_{1}$ können durch die
Tabelle 1. Simulationsergebnisse für $W_{2}=330 \mu \mathrm{m}, V_{o d 1}=0.5 \mathrm{~V}$ und $V_{\text {od } 2}=0.5 \mathrm{~V}$ bei $2.45 \mathrm{GHz}$

\begin{tabular}{lrr}
\hline Parameter & Toolbox & SpectreRF \\
\hline Gain $(\mathrm{dB})$ & 15.2 & 13.7 \\
$\mathrm{HD}_{2}(\mathrm{~dB})$ & -49.2 & -33.2 \\
$\mathrm{HD}_{3}(\mathrm{~dB})$ & -89.9 & -53.9 \\
$\mathrm{IIP}_{2}(\mathrm{dBm})$ & 41.6 & 12.1 \\
$\mathrm{IIP}_{3}(\mathrm{dBm})$ & 14.5 & 0.9 \\
\hline
\end{tabular}

Spezifikationen für die Rauschzahl und die Impedanzanpassung am Eingang und Ausgang festgelegt werden. Hierbei wurde die Dimensionierung nach Lee (2004) durchgeführt. Somit bleiben nur drei Architekturparameter $V_{o d 1}, V_{o d 2}$ und $W_{2}$ unbekannt. Nach dem Einsetzen der Werte aller bekannten Parameter in die Ausdrücke für die Nichtlinearitätsparameter können die graphischen Darstellungen der Nichtlinearitätsparameter durchgeführt werden.

Bei diesem Beispiel wurden zwei Technologieklassen überprüft. Abbildungen 6 und 7 zeigen die graphischen Darstellungen von $\mathrm{HD}_{2}$ und $\mathrm{IM}_{3}$ für die zwei verschiedenen Technologien $(0.25 \mu \mathrm{m}$ und $0.5 \mu \mathrm{m})$ gegen $V_{o d 1}$ und $V_{o d 2}$. Man sieht hier, dass der $0.25 \mu$ Technologieprozess zu einem lineareren Verhalten der Schaltung führt. Abbildungen 8 und 9 zeigen die graphischen Darstellungen von $\mathrm{HD}_{2}$ und $\mathrm{IIP}_{3}$ für den $0.25 \mu \mathrm{m}$ Technologieprozess gegen $W_{2}, V_{\text {od } 1}$ und $V_{\text {od } 2}$. Daraus erkennt man, dass eine Erhöhung der OverdriveSpannung $V_{o d 1}$ und $V_{o d 2}$ zu einer Verbesserung des Verhaltens bzgl. Linearität führt. Dies ist auch zu erwarten, denn die Übertragungskennlinie $\left(I_{D}=f\left(V_{G S}\right)\right)$ des MOSFETs wird mit der Erhöhung der Gate-Source-Spannung linearer. AuBerdem ist zu sehen, dass die Vergrößerung der Kanalbreite des Transistors $M_{2}$ eine positive Auswirkung auf die Linearität der Schaltung hat. Dies ist dadurch zu erklären, dass eine Vergrößerung der Kanalbreite $W_{2}$ zu einer Erhöhung der Kapazität $C_{g s 2}$ führt, was die Rolle eines Filters zwischen dem Transistor $M_{1}$ und $M_{2}$ spielt.

\subsection{Simulation mit SpectreRF}

Um die Ergebnisse der Toolbox zu verifizieren, wurde der LNA im Cadence SpectreRF Design Tool simuliert. SpectreRF ist ein technisch ausgereifter Schaltungssimulator, der einige Analysemethoden einschließlich der Harmonic Balance Methode zur Berechnung der Antworten beim eingeschwungenen Zustand verwendet. Das in der SpectreRF-Simulation verwendete MOSFET-Modell ist ein BSIM3.4 Modell aus der SGB25V Bibliothek der Firma IHP (Frankfurt, Oder).

Die Ergebnisse der SpectreRF-Simulation werden in Tabelle 1 gegen die Ergebnisse der Toolbox aufgelistet. Die Simulationsergebnisse zeigen eine Abweichung zwischen den von der Toolbox ausgerechneten Werten und den Er- 


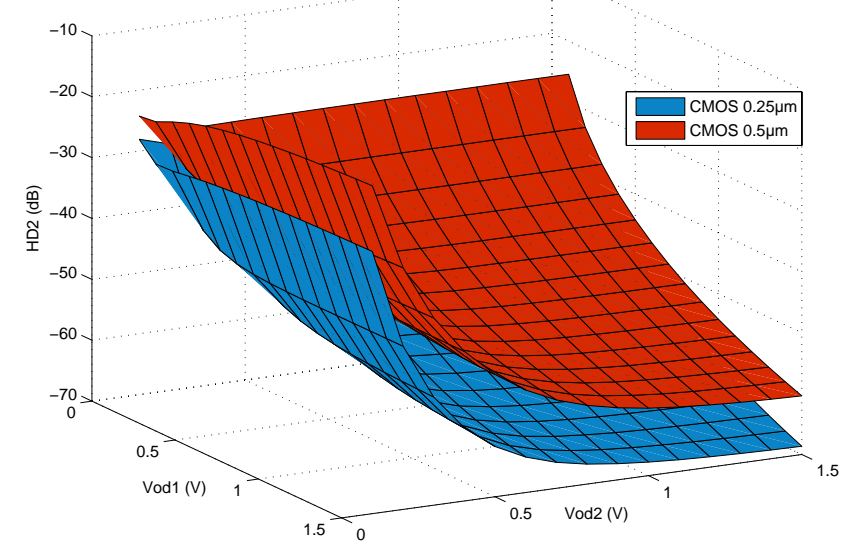

Abb. 6. $\mathrm{HD}_{2}$ für zwei Technologien gegen $V_{o d 1}$ und $V_{o d 2}$.

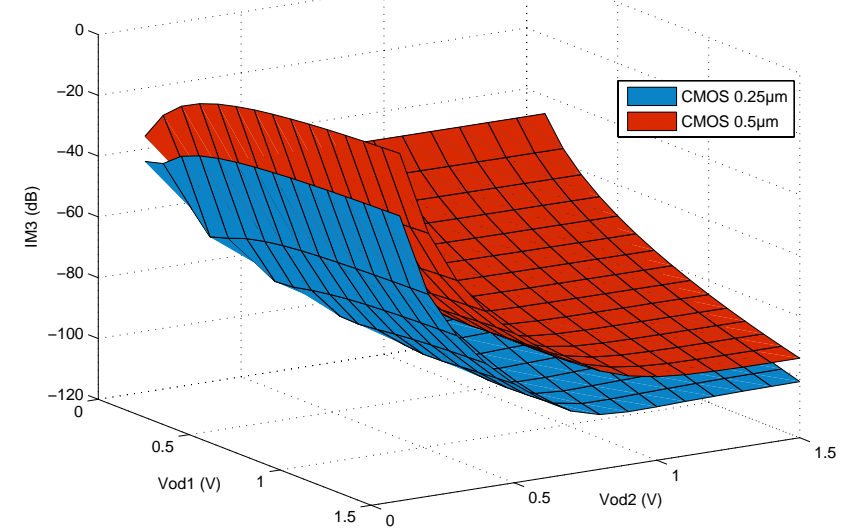

Abb. 7. $\mathrm{IM}_{3}$ für zwei Technologien gegen $V_{o d 1}$ und $V_{o d 2}$.

gebnissen der SpectreRF-Simulation. Die Abweichung ist hauptsächlich aufgrund des großen Unterschieds zwischen dem in der Toolbox verwendeten einfachen MOSFETModell und dem ausgereiften BSIM3.4 Modell in SpectreRF festzustellen. Zweitens ist die Volterraanalyse nur eine Approximationsmethode, was zur Abweichung von den Simulationsergebnissen führt. Trotzdem zeigt der Vergleich mit dem BSIM3.4 Modell eine qualitative Übereinstimmung.

\section{Diskussion}

Eine Prozedur zur Optimierung des Designprozesses für RF Systeme wurde präsentiert. Symbolische Ausdrücke für die Nichtlinearitätsparameter eines Systemblocks wurden mittels Volterra-Reihen in Abhängigkeit von Architekturparametern und Technologieparametern ermittelt. Dies ermöglicht die Beschränkung sowohl der Wertebereiche der

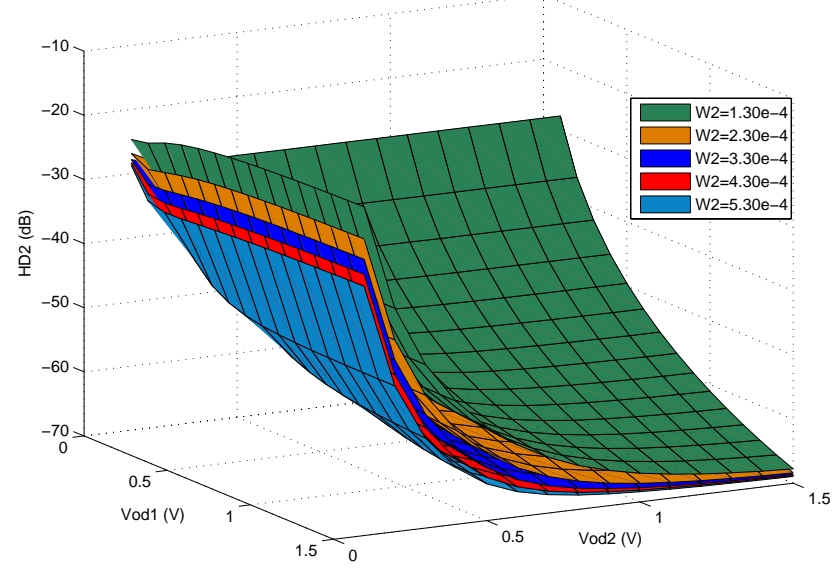

Abb. 8. $\mathrm{HD}_{2}$ für $0,25 \mu \mathrm{m}$ Prozess gegen $W_{2}, V_{o d 1}$ und $V_{o d 2}$.

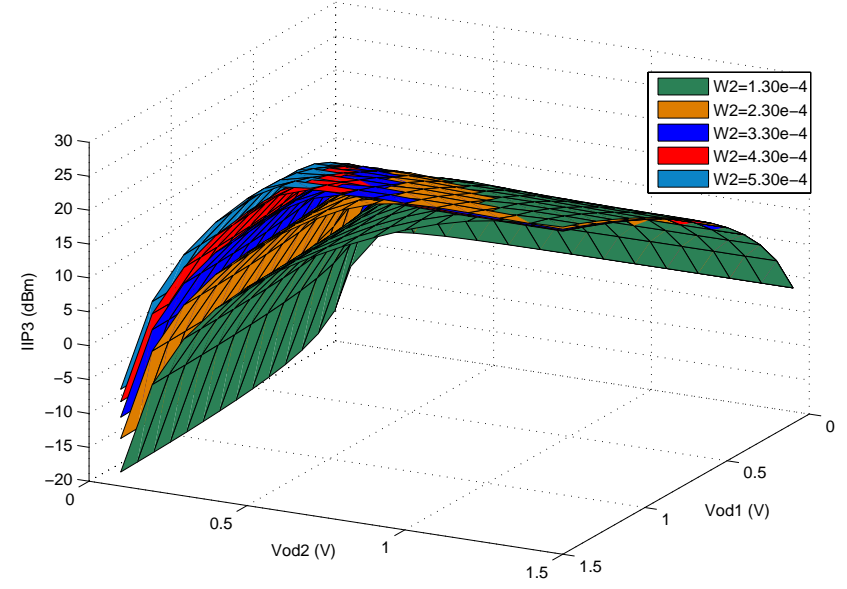

Abb. 9. $\mathrm{IIP}_{3}$ für $0,25 \mu \mathrm{m}$ Prozess gegen $W_{2}, V_{o d 1}$ und $V_{o d 2}$.

Architekturparameter als auch der Klassen der Architekturen und Technologien. Diese Prozedur wurde in einer symbolischen Toolbox implementiert. Ein Vergleich der Ergebnisse aus der Toolbox und der Simulation mit SpectreRF zeigt eine qualitative Übereinstimmung und eine große quantitative Abweichung. Die quantitative Abweichung ist hauptsächlich aufgrund des großen Unterschieds zwischen dem in der Toolbox verwendeten einfachen MOSFET-Modell und dem ausgereiften BSIM3.4 Modell in SpectreRF festzustellen. Zweitens ist die Volterraanalyse nur eine Approximationsmethode, was zur Abweichung von den Simulationsergebnissen führt. In einer zukünftigen Arbeit kann ein exakteres MOSFET-Modell z.B. das EKV-Modell (Enz et al., 1995) in der Toolbox verwendet werden, um quantitativ realistischere Ergebnisse zu erzeugen. 


\section{Literatur}

Enz, C. C., Krummenacher, F., und Vittoz, E. A.: An analytical MOS Transistor model valid in all regions of operation and dedicated to low-voltage and low-current applications, Analoge integrated circuits and signal processing journal on low-voltage and low-power design, 83-114, 1995.

Gielen, G. und Sansen, W.: Symbolic analysis for automated design of analog integrated circuits, Kluwer Academic Publishers, 115$117,1991$.

Gielen, G., Walscharts, H., und Sansen, W.: ISAAC: a symbolic simulator for analog integrated circuits, IEEE J. Solid-St. Circ., 24(6), 1587-1597, December 1989.
Lee, T.: The Design of CMOS Radio-Frequency Integrated Circuits, Cambridge University Press, Cambridge, 364-384, 2004.

Razavi, B.: Design of analog CMOS integrated circuits, McGrawHill International Editions, 588-589, 2001.

Schetzen, M.: The Volterra and Wiener Theories of Nonlinear Systems, Wiley-Interscience, 7 pp., 1980.

Wambcaq, P. und Sansen, W.: Distortion Analysis of Analog Integrated Circuits, Kluwer Academic Publishers, 77, 119-128, 1998.

Zadeh, L. A.: A contribution to the theory of nonlinear systems, J. Franklin Inst., 255(5), 387-408, 1953. 